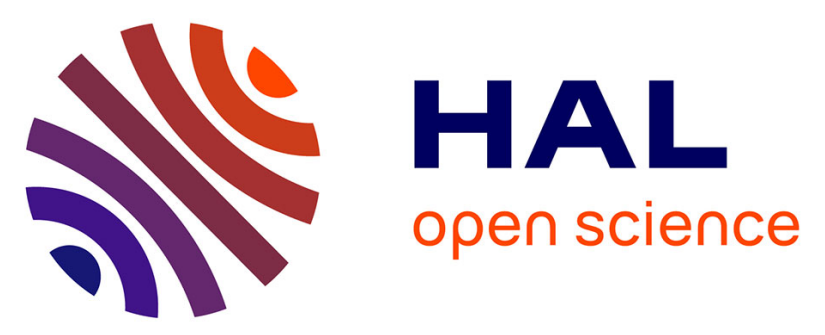

\title{
Third-order structure functions in rotating and stratified turbulence: a comparison between numerical, analytical and observational results
}

Enrico Deusebio, Pierre Augier, Erik Lindborg

\section{- To cite this version:}

Enrico Deusebio, Pierre Augier, Erik Lindborg. Third-order structure functions in rotating and stratified turbulence: a comparison between numerical, analytical and observational results. Journal of Fluid Mechanics, 2014, 755, pp.294-313. 10.1017/jfm.2014.414 . hal-01392587

\author{
HAL Id: hal-01392587 \\ https://hal.science/hal-01392587
}

Submitted on 30 Oct 2018

HAL is a multi-disciplinary open access archive for the deposit and dissemination of scientific research documents, whether they are published or not. The documents may come from teaching and research institutions in France or abroad, or from public or private research centers.
L'archive ouverte pluridisciplinaire HAL, est destinée au dépôt et à la diffusion de documents scientifiques de niveau recherche, publiés ou non, émanant des établissements d'enseignement et de recherche français ou étrangers, des laboratoires publics ou privés. 


\title{
Third-order structure functions in rotating and stratified turbulence: a comparison between numerical, analytical and observational results
}

\author{
Enrico Deusebio $^{1,2, \dagger}$, P. Augier ${ }^{1,2}$ and E. Lindborg ${ }^{2}$ \\ ${ }^{1}$ Department of Applied Mathematics and Theoretical Physics, Centre for Mathematical Sciences, \\ University of Cambridge, Wilberforce Road, Cambridge CB3 0WA, UK \\ ${ }^{2}$ Linné Flow Centre, Department of Mechanics, Royal Institute of Technology (KTH), \\ SE-10044 Stockholm, Sweden
}

First, we review analytical and observational studies on third-order structure functions including velocity and buoyancy increments in rotating and stratified turbulence and discuss how these functions can be used in order to estimate the flux of energy through different scales in a turbulent cascade. In particular, we suggest that the negative third-order velocity-temperature-temperature structure function that was measured by Lindborg \& Cho (Phys. Rev. Lett., vol. 85, 2000, p. 5663) using stratospheric aircraft data may be used in order to estimate the downscale flux of available potential energy (APE) through the mesoscales. Then, we calculate third-order structure functions from idealized simulations of forced stratified and rotating turbulence and compare with mesoscale results from the lower stratosphere. In the range of scales with a downscale energy cascade of kinetic energy (KE) and APE we find that the third-order structure functions display a negative linear dependence on separation distance $r$, in agreement with observation and supporting the interpretation of the stratospheric data as evidence of a downscale energy cascade. The spectral flux of APE can be estimated from the relevant third-order structure function. However, while the sign of the spectral flux of KE is correctly predicted by using the longitudinal third-order structure functions, its magnitude is overestimated by a factor of two. We also evaluate the third-order velocity structure functions that are not parity invariant and therefore display a cyclonic-anticyclonic asymmetry. In agreement with the results from the stratosphere, we find that these functions have an approximate $r^{2}$-dependence, with strong dominance of cyclonic motions.

Key words: atmospheric flows, turbulent flows, turbulence theory

\section{Introduction}

Statistical analysis of atmospheric measurements show that horizontal spectra of both wind and temperature in the upper troposphere and lower stratosphere follow a $k^{-5 / 3}$ power law over more than two decades, at wavenumbers corresponding to

$\dagger$ Email address for correspondence: deusebio@mech.kth.se 
wavelengths between 2 and $500 \mathrm{~km}$ (Nastrom \& Gage 1985). Such a power law is a strong indication that there is an energy cascade, that is a systematic transfer of energy between different scales by nonlinear interactions. Either there is positive spectral kinetic energy (KE) flux, $\Pi_{K}$, which means that energy flows from small to large wavenumbers, as in the downscale energy cascade of three-dimensional isotropic turbulence (Kolmogorov 1941b), or there is a negative spectral energy flux, which means that energy flows in the opposite direction, as in the upscale energy cascade of two-dimensional turbulence (Kraichnan 1967) or quasi-geostrophic (QG) turbulence (Charney 1971). A measurement of the energy spectrum is not sufficient to discriminate between these two types of cascades since they both exhibit an energy spectrum of the form $E(k) \sim\left|\Pi_{K}\right|^{2 / 3} k^{-5 / 3}$. Dewan (1979) hypothesized that the dynamics producing the mesoscale $k^{-5 / 3}$ power law in the upper troposphere and lower stratosphere is a downscale energy cascade of interacting gravity waves, while Lilly (1983) hypothesized that the dynamics is an upscale energy cascade of layerwise two-dimensional turbulence. Lindborg (1999) suggested that measurements of third-order velocity structure functions could give an answer to the question regarding the direction of the energy cascade. To obtain such an answer one should use a relation which is of similar form to the so-called 'four-fifths law' of isotropic three-dimensional turbulence (Kolmogorov 1941a)

$$
\left\langle\delta v_{L} \delta v_{L} \delta v_{L}\right\rangle=-\frac{4}{5} \Pi_{K} r
$$

where $\langle\cdot\rangle$ denotes averaging and $\delta v_{L}(\boldsymbol{r})=v_{L}^{\prime}-v_{L}$ is the difference between the longitudinal velocity component at two points separated by a vector $\boldsymbol{r}$, where the longitudinal component is the projection onto the unit vector $\boldsymbol{n}=\boldsymbol{r} / r$. The mean energy dissipation per unit mass, $\varepsilon$, may be substituted for $\Pi_{K}$ in (1.1), since they are equal when the cascade is downscale.

The four-fifths law is the three-dimensional incompressible and isotropic solution to a more general flux relation which can be written as

$$
\nabla \cdot J_{K}=-4 \Pi_{K}
$$

where $\boldsymbol{J}_{K}=\langle\delta \boldsymbol{v} \delta \boldsymbol{v} \cdot \delta \boldsymbol{v}\rangle$. The two-dimensional isotropic solution to this relation can be written as (Lindborg 1999)

$$
\left\langle\delta v_{L} \delta v_{L} \delta v_{L}\right\rangle+\left\langle\delta v_{L} \delta v_{T} \delta v_{T}\right\rangle=-2 \Pi_{K} r
$$

where $\delta v_{T}$ is the transverse velocity increment, that is an increment of a velocity component which is perpendicular to $\boldsymbol{n}$. By incompressibility one can also derive the relation

$$
\left\langle\delta v_{L} \delta v_{L} \delta v_{L}\right\rangle=-\frac{3}{2} \Pi_{K} r
$$

for two-dimensional turbulence. Comparing the three-dimensional relation (1.1) with the two-dimensional relations (1.3) and (1.4) we see that they have the same form. The third-order structure function is proportional to the spectral energy flux and is linear in the separation distance. However, there is a very important difference. In three-dimensional turbulence the spectral energy flux is positive while it is negative in two-dimensional turbulence. Therefore, an upscale energy cascade in the mesoscales would mean that the third-order structure function should display a positive linear dependence on $r$. On the other hand, a negative linear dependence on $r$ would be an indication of a downscale energy cascade. Cho \& Lindborg (2001) measured the third-order structure function in the lower stratosphere, with $r$ lying in the horizontal 


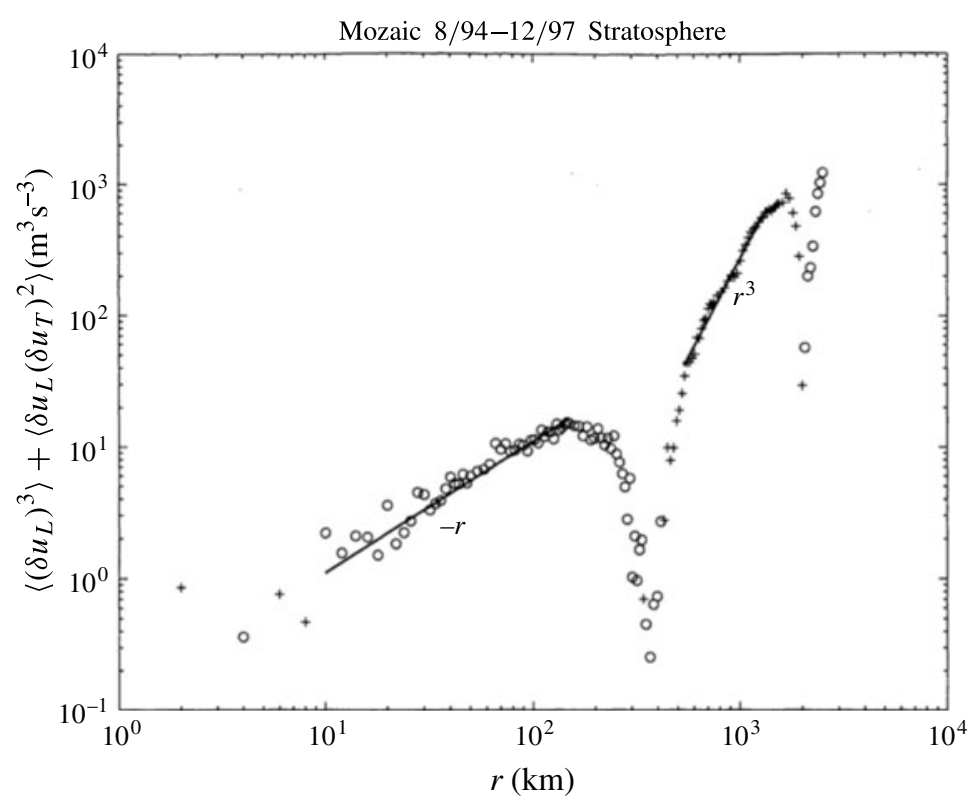

FIgURE 1. Sum of the diagonal third-order structure functions. Straight lines indicate the power laws $-r$ and $r^{3}$. Circles and crosses indicate negative and positive values, respectively. (Reproduced from Cho \& Lindborg 2001.)

plane (constant-pressure surfaces), using a huge amount of aircraft data. In figure 1 we show the function $\left\langle\delta v_{L} \delta v_{L} \delta v_{L}\right\rangle+\left\langle\delta v_{L} \delta v_{T} \delta v_{T}\right\rangle$ plotted versus separation distance $r$. In the interval $10 \mathrm{~km}<r<150 \mathrm{~km}$ the third-order structure function exhibits a negative linear range. At $r \approx 400 \mathrm{~km}$ there is a switch of sign and the third-order structure function is clearly positive at scales of the order of $1000 \mathrm{~km}$. Cho \& Lindborg (2001) interpreted the negative linear dependence on $r$ as a sign of a downscale energy cascade and used the two-dimensional isotropic relation (1.3) to estimate the positive $\mathrm{KE}$ flux as $\Pi_{K} \approx 6 \times 10^{-5} \mathrm{~m}^{2} \mathrm{~s}^{-3}$. It can be questioned if it is correct to use the two-dimensional relation to estimate $\Pi_{K}$, since a strictly two-dimensional dynamics would, rather, give a negative $\Pi_{K}$.

Another complication is that in general there can be a double cascade of available potential energy (APE, Lorenz 1955) and KE. In a linearly-stratified flow, there are in fact two flux relations (Augier, Galtier \& Billant 2012)

$$
\begin{aligned}
& \nabla \cdot J_{K}=-4 \Pi_{K}+2\langle\delta b \delta w\rangle, \\
& \nabla \cdot J_{P}=-4 \Pi_{P}-2\langle\delta b \delta w\rangle .
\end{aligned}
$$

Here, $b=\theta^{\prime} g / \theta_{0}$ is the buoyancy, $\theta^{\prime}$ and $\theta_{0}$ are the fluctuating and mean potential temperature respectively, $g$ is the acceleration due to gravity, $w$ is the vertical velocity, $\Pi_{P}$ is the spectral flux of APE and $\boldsymbol{J}_{P}=\langle\delta \boldsymbol{v} \delta b \delta b\rangle / N^{2}$, where $N$ is the Brunt-Väisälä frequency. The last term in (1.5), which appears with opposite sign in (1.6), represents energy exchange between KE and APE. In a range of scales where there is a selfsimilar energy cascade of universal character the ratio between KE and APE should be scale independent and this term should therefore be negligible. Neglecting this term and introducing the assumption of axisymmetry the relations (1.5) and (1.6) can be 


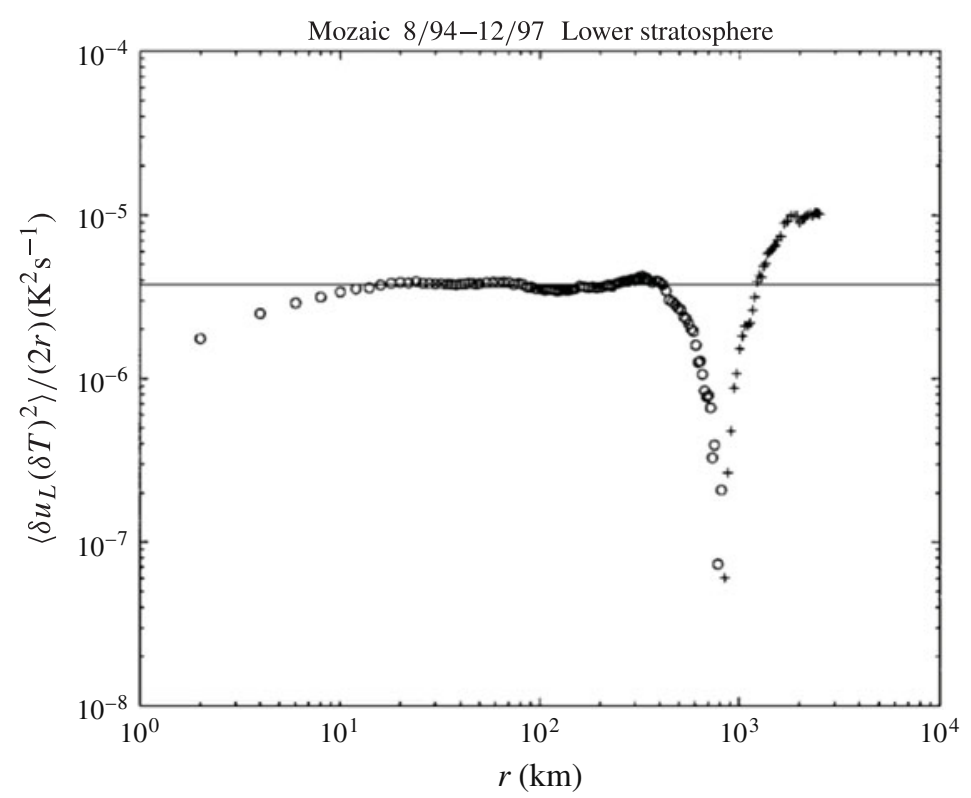

FIgURE 2. Plot of $\left\langle\delta u_{T} \delta T \delta T\right\rangle /(2 r)$ versus $r$. Solid line: best linear fit, $20 \mathrm{~km}<r$ $<400 \mathrm{~km}$. Circles and crosses indicate negative and positive values, respectively. (Reproduced from Lindborg \& Cho 2000.)

written as

$$
\begin{aligned}
\frac{1}{r_{h}} \frac{\partial}{\partial r_{h}}\left(r_{h}\left\langle\delta u_{L} \delta \boldsymbol{v} \cdot \delta \boldsymbol{v}\right\rangle\right)+\frac{\partial}{\partial r_{z}}\langle\delta w \delta \boldsymbol{v} \cdot \delta \boldsymbol{v}\rangle & =-4 \Pi_{K}, \\
\frac{1}{r_{h}} \frac{\partial}{\partial r_{h}}\left(r_{h}\left\langle\delta u_{L} \delta b \delta b\right\rangle / N^{2}\right)+\frac{\partial}{\partial r_{z}}\langle\delta w \delta b \delta b\rangle / N^{2} & =-4 \Pi_{P},
\end{aligned}
$$

where $r_{h}$ is the radial coordinate, that is the projection of $\boldsymbol{r}$ on the horizontal plane, $r_{z}$ is the axial coordinate and the velocity is decomposed as $\boldsymbol{v}=\boldsymbol{u}+w \boldsymbol{e}$, with $\boldsymbol{u}=u_{L} \boldsymbol{n}+u_{T} \boldsymbol{t}$ the horizontal velocity. Here, $\boldsymbol{t}$ and $\boldsymbol{e}$ are the azimuthal and axial unit vectors, respectively. Taking $\boldsymbol{r}$ to be in the horizontal plane and assuming that all terms including the vertical velocity can be neglected these relations can be integrated to give

$$
\begin{aligned}
\left\langle\delta u_{L} \delta u_{L} \delta u_{L}\right\rangle+\left\langle\delta u_{L} \delta u_{T} \delta u_{T}\right\rangle & =-2 \Pi_{K} r \\
\left\langle\delta u_{L} \delta b \delta b\right\rangle / N^{2} & =-2 \Pi_{P} r
\end{aligned}
$$

where we have changed the name of the radial coordinate from $r_{h}$ to $r$, which is natural when $\boldsymbol{r}$ lies in the horizontal plane. The relation (1.9) is identical to the twodimensional relation (1.3) which was used by Cho \& Lindborg (2001).

Lindborg \& Cho (2000) also calculated the third-order velocity-temperaturetemperature structure function, $\left\langle\delta u_{L} \delta T \delta T\right\rangle$. In figure 2 we show this function divided by $2 r$, plotted versus separation distance. In the interval $20 \mathrm{~km}<r<400 \mathrm{~km}$ there is a range where $\left\langle\delta u_{L} \delta T \delta T\right\rangle /(2 r)$ is negative and constant. In fact, this result is much cleaner than the corresponding result for the velocity structure function seen in figure 1 , and the range with a negative linear dependence is much wider. We will now interpret this result in the light of relation (1.10), an interpretation that was not 
given by Lindborg \& Cho (2000). The aircraft data are taken at constant-pressure surfaces. Therefore, changes in the potential temperature $\theta$ are only due to changes in absolute temperature $T$. On a such surface we therefore have

$$
\left\langle\delta u_{L} \delta b \delta b\right\rangle=\frac{\left\langle\delta u_{L} \delta T \delta T\right\rangle g^{2}}{T_{0}^{2}},
$$

where $T_{0}$ is the mean temperature. Using relations (1.10) and (1.11), the typical lower stratospheric values $N^{2}=4 \times 10^{4} \mathrm{~s}^{-2}$ and $T_{0}=220 \mathrm{~K}$ (Charney \& Drazin 1961), and the constant value $3.9 \times 10^{-6} \mathrm{~K}^{2} \mathrm{~s}^{-1}$ from figure 2, we can estimate the spectral flux of APE as

$$
\Pi_{P} \approx 2 \times 10^{-5} \mathrm{~m}^{2} \mathrm{~s}^{-3} .
$$

With the estimated value of $\Pi_{K}$ we would have $\Pi_{K} / \Pi_{P} \approx 3$.

The interpretation of the negative linear range of the third-order structure functions from the lower stratosphere, seen in figures 1 and 2, as evidence of a downscale energy cascade has gained considerable support from theoretical and numerical advances in strongly stably stratified dynamics, although recent experiments on electromagnetically forced turbulence in rotating thick layers have also revived the hypothesis of an upscale energy transfer (Xia et al. 2011). Both scaling analysis (Billant \& Chomaz 2001; Lindborg 2006; Brethouwer et al. 2007), idealized numerical simulations (Laval, McWilliams \& Dubrulle 2003; Riley \& deBruynKops 2003; Waite \& Bartello 2004; Lindborg 2006; Brethouwer et al. 2007) and experiments (Augier, Billant \& Chomaz 2013; Augier et al. 2014) show that there exists a strongly stratified turbulent regime at horizontal scales which are considerably larger than the Ozmidov length scale, $l_{0}=\varepsilon^{1 / 2} / N^{3 / 2}$. The conditions for such a regime to exist are that the horizontal Froude number $F_{h}=U /(L N)$, where $U$ is a characteristic horizontal velocity and $L$ a characteristic horizontal length scale, is small and that the buoyancy Reynolds number $\operatorname{Re}=\varepsilon /\left(v N^{2}\right)$, where $v$ is the kinematic viscosity, is large. Due to the strong intermittency of stratified turbulence, there is a strong variation of the Ozmidov length scale in the lower stratosphere. A typical value can be estimated to be around $10 \mathrm{~m}$ (Lindborg 2006). Classical isotropic turbulence, where horizontal and vertical velocity fluctuations are of the same order of magnitude, can be observed at scales below the Ozmidov length scale. At larger scales, vertical velocity fluctuations are strongly damped by buoyancy forces.

Yet, in this regime there exists a turbulent energy cascade which is not strictly twodimensional, as initially suggested by Lilly (1983), since the nonlinear advection terms in the momentum equation including the vertical velocity are of the same order of magnitude as the corresponding horizontal advection terms, that is

$$
\left|w \partial_{z} u\right| \sim\left|u \partial_{x} u\right|,
$$

where $x$ is a horizontal coordinate and $u$ the corresponding horizontal velocity component. The reason why this can be true is that the vertical length scale of the turbulent motions decreases with increased degree of stratification, so that $\left|\partial_{z} u\right|$ increases at the same rate as $|w|$ decreases when the stratification becomes strong. In this regime, there is a downscale energy cascade of $\mathrm{KE}$, which is totally dominated by the horizontal velocity component, as well as APE. The estimate $\Pi_{K} / \Pi_{P} \approx 3$ which we have made on the basis of (1.9), (1.10) and the stratospheric data presented in figures 1 and 2 is consistent with results from numerical simulations of stratified turbulence (Lindborg 2006). It has also been demonstrated that the strongly stratified 
turbulence regime can be obtained in the presence of system rotation (Lindborg 2005; Waite \& Bartello 2006), provided that the rotation is not too strong.

Recently, Vallgren, Deusebio \& Lindborg (2011) and Deusebio, Vallgren \& Lindborg (2013) carried out numerical simulations of strongly stratified turbulence at strong system rotation, that is at small Rossby number $R o=U / f L$, where $f$ is the Coriolis parameter. They showed that even for very small Ro a substantial part of the injected energy cascades towards small scales. The amount of energy going downscale increases with $R o$, i.e. as the rotation rate decreases. This leakage of energy from the large-scale QG flow leads at smaller scales to a strongly stratified turbulent regime with a downscale cascade of KE and APE, displaying $k^{-5 / 3}$ energy spectra. At large scales, however, there is a QG regime displaying a $k^{-3}$ energy spectrum. The superposition of the $k^{-5 / 3}$ energy spectrum of stratified turbulence with the $k^{-3}$ spectrum of QG turbulence gives a compound spectrum which is similar to that measured by Nastrom \& Gage (1985) in the atmosphere.

An issue which is still debated is what role gravity waves have in the downscale energy cascade, producing the Nastrom-Gage spectrum. As stated previously, it was hypothesized by Dewan (1979) that the downscale cascade is a process in which gravity waves are successively transferring their energy to modes with shorter wavelengths, by nonlinear interaction. Bartello (1995) argued that the most important triad interaction transferring energy downscale is the type where two wave modes interact with a vortical mode, with the last one acting as catalyst. This picture has recently been advanced by Waite \& Snyder (2009) and Waite \& Snyder (2013) who carried out simulations of an idealized baroclinic wave cycle, showing the spontaneous development of gravity waves which caused the shallowing of the spectrum. On the other hand, it has also been argued that waves play a minor role in the downscale energy cascade. Lindborg (2007) used structure functions calculated from aircraft data to make a rotational-divergent decomposition of the energy spectrum and found that rotational energy is somewhat larger than divergent energy, from which it can be argued that gravity waves cannot be the only source of energy in the $k^{-5 / 3}$-spectrum. Moreover, Deusebio et al. (2013) found some evidence suggesting that resonant wave interactions cannot explain the downscale cascade of energy of their turbulent simulations in systems close to geostrophy. In this study, however, we will not investigate this issue further.

In this study we calculate third-order structure functions from simulations of forced stratified rotating turbulence and compare the results from the simulations with the stratospheric data and the different analytical relations which we have reviewed in this introduction. In particular, we will focus on whether it is correct to make the approximation that are needed in order to derive (1.9) and (1.10). In addition to this, we will also investigate if our simulations are in agreement with another interesting observation made by Cho \& Lindborg (2001) regarding the third-order horizontal velocity structure functions that are not parity invariant, that are the functions $\left\langle\delta u_{T} \delta u_{T} \delta u_{T}\right\rangle$ and $\left\langle\delta u_{T} \delta u_{L} \delta u_{L}\right\rangle$, where $\delta u_{T}$ is the azimuthal velocity increment in the cyclonic direction. In the absence of system rotation parity invariance should hold and in this case these functions must be zero. In the presence of system rotation parity invariance is broken and there may be a cyclonic-anticyclonic asymmetry. Cho \& Lindborg (2001) and Lindborg \& Cho (2001) found that both these functions are positive in the lower stratosphere and display a clean $r^{2}$-dependence in the interval $10 \mathrm{~km}<r<1000 \mathrm{~km}$. That these functions are positive means that cyclonic motions are dominant. However, no theoretical explanation can yet be offered for the $r^{2}$-dependence. 
The paper is organized as follows. In $\S 2$, we briefly present the simulations. In $\S 3$, we present the results for the third-order structure functions. Second-order statistics, such as energy spectra, have already been presented in Deusebio et al. (2013). In this paper, we therefore focus on the third-order structure functions. In $\S 4$, we end the paper with conclusions.

\section{Simulations}

The simulations are standard box simulations of the Boussinesq equations, using triply periodic boundary conditions and white noise (in time) forcing in a limited wavenumber band (Deusebio et al. 2013). The equations are formulated and solved for the three variables, $q, a_{1}$ and $a_{2}$, where

$$
q=-\frac{\partial u}{\partial y}+\frac{\partial v}{\partial x}+\frac{f}{N^{2}} \frac{\partial b}{\partial z},
$$

is the Charney potential vorticity (Charney 1971), and

$$
\begin{aligned}
& a_{1}=-\frac{f}{N} \frac{\partial v}{\partial z}+\frac{1}{N} \frac{\partial b}{\partial x} \\
& a_{2}=\frac{f}{N} \frac{\partial u}{\partial z}+\frac{1}{N} \frac{\partial b}{\partial y}
\end{aligned}
$$

are two 'ageostrophic components' that measure the departure from thermal wind balance (Vallis 2006). The formulation using $q, a_{1}$ and $a_{2}$ is similar to the normal modes decomposition (see e.g. Bartello 1995; Embid \& Majda 1998; Smith \& Waleffe 2002). Here $u, v$ and $w$ are the velocity components in the $x, y$ and $z$ directions, respectively, where $x, y$ are the horizontal coordinates, $z$ the vertical coordinate and $b$ is the buoyancy. For the explicit form of the equations that are solved, the reader is referred to Deusebio et al. (2013). The equations contain two dimensionless parameters, the Froude number and the Rossby number, which are defined as

$$
F r=\frac{U}{N L}, \quad R o=\frac{U}{f L},
$$

where $U$ is a characteristic horizontal velocity, $N$ the Brunt-Väisalä frequency, $f$ the Coriolis parameter and $L$ a characteristic horizontal length scale.

The system of equations is discretized in a triply periodic domain $\left(L_{x}, L_{y}, L_{z}\right)=$ $(2 \pi, 2 \pi, 2 \pi)$, using QG scaling (Charney 1971), which means that the vertical coordinate has been stretched by a factor of $N / f$. The periodicity in all the three directions allows a Fourier representation along $x, y$ and $z ; N_{x}=N_{y}=N_{z}=1024$ modes were used in each direction. Pseudo-spectral methods are employed and the equations are advanced in time in spectral space. The nonlinear terms are evaluated in physical space and are discretized in time using a low-storage fourth-order Runge-Kutta scheme. In order to prevent aliasing errors, the 2/3-dealiasing rule was applied to the nonlinear terms. Linear terms are instead separately solved using an exact implicit procedure (Canuto et al. 1988).

Two different forcing schemes are adopted. In the first, which we call $Q$-forcing, the forcing is restricted to the $q$-equation. This is the same scheme as used in Vallgren et al. (2011) and Deusebio et al. (2013). In the second scheme, which we call mixed 


$\begin{array}{lccc}\text { Run } & R o & k_{f} & \varepsilon / P \\ \text { Q2 } & 0.2 & 4 & 0.27 \\ \text { Q1 } & 0.1 & 4 & 0.08 \\ \text { Q05 } & 0.05 & 4 & 5.9 \times 10^{-3} \\ \text { Q1-SS } & 0.1 & 54 & 0.08 \\ \text { M2 } & 0.2 & 4 & 0.88 \\ \text { M1 } & 0.1 & 4 & 0.78 \\ \text { M05 } & 0.05 & 4 & 0.69\end{array}$

TABLE 1. Summary of the simulations. The last column shows the ratio between small-scale dissipation, $\varepsilon$, and energy injection, $P$, in the quasi-stationary state.

forcing or $M$-forcing, the forcing is applied to all three equations, with about twothirds of the energy injection into the ageostrophic modes, $a_{1}$ and $a_{2}$. This scheme is similar to that used in a number of studies in stratified turbulence (Brethouwer et al. 2007; Lindborg \& Brethouwer 2007). In both cases energy is injected in a limited wavenumber band in the vertically stretched wavenumber space, $k \in\left[k_{f}-\Delta k / 2, k_{f}+\right.$ $\Delta k / 2]$, where $\Delta k=2$. The characteristic length scale of the forcing is therefore $L_{f}=$ $2 \pi / k_{f}$. As summarized in table 1 we have carried out three simulations at different $R o$ with large-scale $\left(k_{f}=4\right) Q$-forcing, three simulations at different $R o$ with large-scale $M$-forcing and one simulation with $Q$-forcing at an intermediate wavenumber $\left(k_{f}=54\right)$. In all simulations the Froude number is equal to 0.01 .

Energy is transferred both upscale and downscale, as compared to the forcing scale, with the ratio of energy cascading upscale and downscale mainly controlled by Ro (Deusebio et al. 2013). It is worth noting that the ratio between the amount of energy cascading downscale and upscale is not a universal function and considerable differences are found between the $Q$ - and the $M$-runs. Nevertheless, the same trend is observed, i.e. the ratio decreases with decreasing $R o$. That $M$-runs have larger small-scale dissipation is also consistent with the analysis of Bartello (1995) and the fact that ageostrophic energy (directly forced in the $M$-runs), by not being constrained by potential vorticity conservation, cascades directly to small scales. A large-scale linear drag as well as hyperviscosity (using a Laplacian to the power of four in a stretched grid) are introduced in the flow in order to allow for energy dissipation at large and small scales, respectively. After an initial transient, an approximate statistically stationary state develops from which statistics are collected.

\section{Results}

In figure 3 we plot the total spectral energy flux for the two runs Q2 and Q1. The curves are normalized with the total energy injection rate $P$. Part of the energy which is injected is going into an upscale energy cascade, which is seen as a negative flux at smaller wavenumbers than the forcing wavenumber. The remaining part is going into a downscale energy cascade which is seen as a positive flux at larger wavenumbers than the forcing wavenumber. As shown by Vallgren et al. (2011), the fraction of energy which is going downscale increases with $R o$ which is also illustrated in figure 1 . The total spectral flux can be divided into the flux of $\mathrm{KE}, \Pi_{K}$, and the flux of $\mathrm{APE}, \Pi_{P}$. There is a range where the fluxes are almost constant. We determine the spectral fluxes, $\Pi_{K}$ and $\Pi_{P}$, by taking an average over $k \in[15,50]$. 


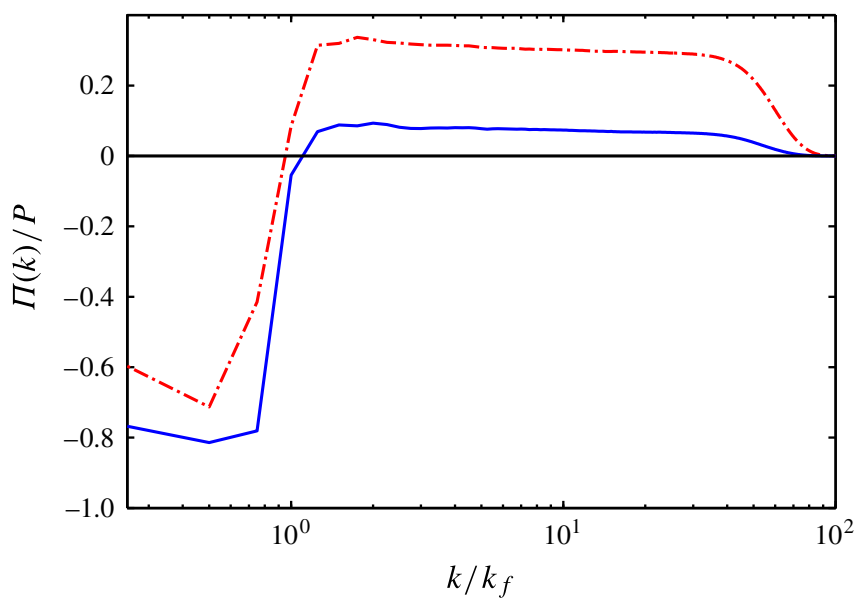

FIgURE 3. (Colour online) Total energy flux normalized with the total energy input $P$ for $\longrightarrow, \mathrm{Q} 1(R o=0.1)$ and $-\cdot-, \mathrm{Q} 2(R o=0.2)$. The forcing scale is at $k_{f}=4$.

In figure 4 we plot the function

$$
-\frac{\left\langle\delta u_{L} \delta \boldsymbol{u} \cdot \delta \boldsymbol{u}\right\rangle}{2 \Pi_{K} r}=-\frac{\left\langle\delta u_{L} \delta u_{L} \delta u_{L}\right\rangle+\left\langle\delta u_{L} \delta u_{T} \delta u_{T}\right\rangle}{2 \Pi_{K} r}
$$

for the six simulations with large-scale forcing. This function should be positive and equal to unity in a range where $\Pi_{K}$ is positive and where the relation (1.9) is satisfied. Indeed, all curves are positive in the relevant range of separation distances. If we used the curves in order to determine the sign of $\Pi_{K}$ we would therefore obtain the correct answer in all the cases. However, the curves generally overshoot unity. In all cases we would overestimate the magnitude of $\Pi_{K}$ by a factor of approximately 2 if we used relation (1.9) in a similar way as done by Cho \& Lindborg (2001) for the third-order structure functions seen in figure 1 .

In figure 5 we plot the function

$$
-\frac{\left\langle\delta u_{L} \delta b \delta b\right\rangle}{2 N^{2} \Pi_{P} r}
$$

for the same runs as in figure 4. For all curves there is a range where this function is reasonably close to unity, in agreement with the relation (1.10). For the simulation with $Q$-forcing at the lowest Rossby number, $R o=0.05$, this range is very narrow. However, at small separation distances the curve from this simulation collapses fairly well on the other curves. Thus, the results from the simulations support the interpretation of figure 2 as giving evidence of a positive or downscale energy flux of APE through the mesoscales, whose magnitude can be estimated by using the relation (1.10).

In figure 6 we plot $\boldsymbol{\nabla} \cdot \boldsymbol{J}_{K}$ and $\boldsymbol{\nabla} \cdot \boldsymbol{J}_{P}$ from run Q1. We also show the separate contributions from the horizontal divergence and the vertical divergence corresponding to the terms on the left-hand sides of (1.7) and (1.8). In addition, we show the energy exchange term $2\langle\delta w \delta b\rangle$ which appear with different signs on the right-hand sides of (1.5) and (1.6). In figure 6(a) we plot the terms from the KE flux relation normalized by $\Pi_{K}$ and in figure $6(b)$ we plot the terms in the potential energy flux relation 


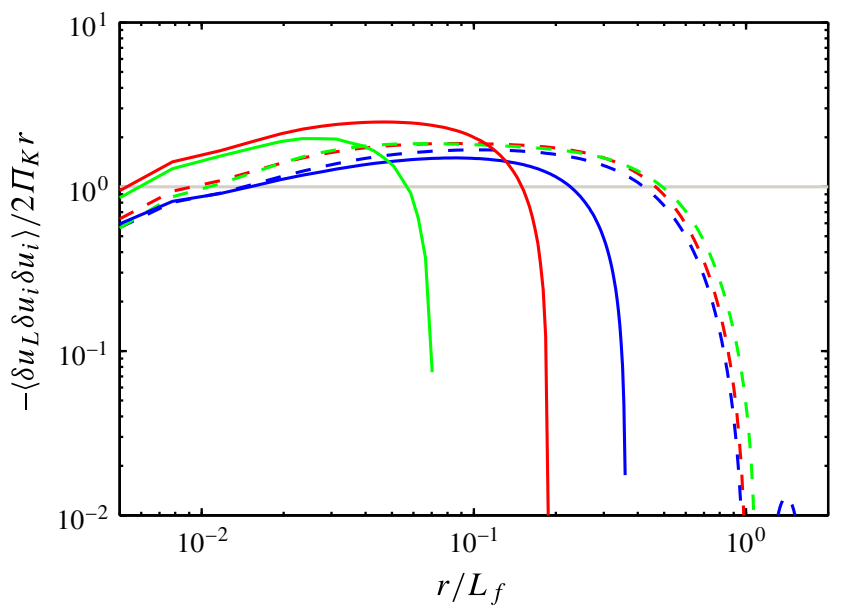

FIGURE 4. Plot of $-\left\langle\delta u_{L} \delta \boldsymbol{u} \cdot \delta \boldsymbol{u}\right\rangle / 2 \Pi_{K} r$ for the different runs. Solid lines: $Q$-forcing; dashed lines: $M$-forcing. Green $R o=0.05$; red $R o=0.1$; blue $R o=0.2$.

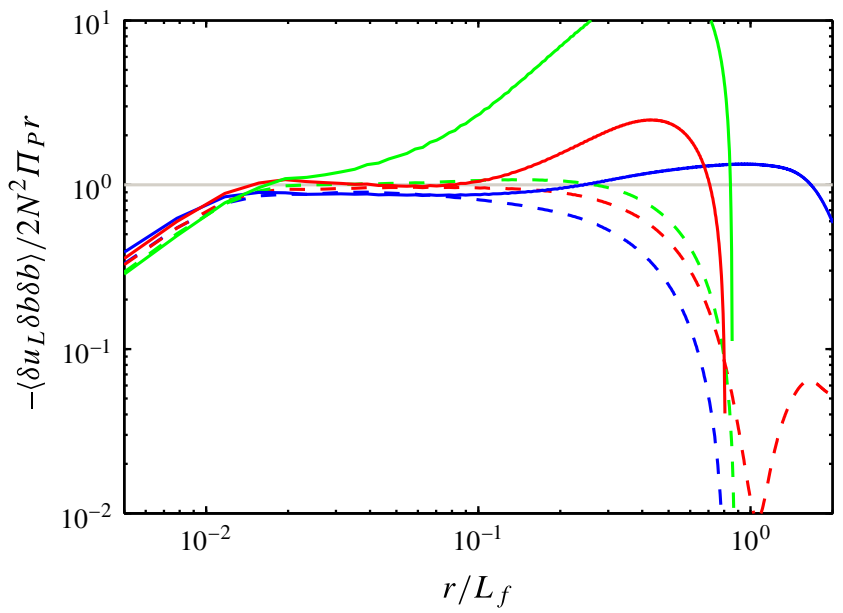

FIGURE 5. Plot of $-\left\langle\delta u_{L} \delta b \delta b\right\rangle / 2 N^{2} \Pi_{P} r$ for the different runs. Lines as in figure 4.

normalized by $\Pi_{P}$. The energy exchange term is small at small separations and in both figures there is a narrow range where the total normalized flux is equal to -4 , confirming the theoretical relations (1.7) and (1.8). For the KE flux, the contribution from the vertical part of the divergence is half that from the horizontal divergence, but with opposite sign. This is the reason why the curves in figure 4 overshoot unity. On the other hand, for the APE flux, the contribution from the vertical part of the divergence is small and the total divergence can therefore be approximated by the horizontal part. This is the reason why the curves in figure 5 are all close to unity.

We find it somewhat unexpected that the vertical divergence makes such a significant contribution to the total divergence and has opposite sign to the horizontal divergence. Figure 7 shows the ratio (in absolute value) of the vertical and horizontal contributions to the total divergence for both $Q$ - and $M$-runs. We have also carried out two additional simulations in order to check the convergence of the results with 

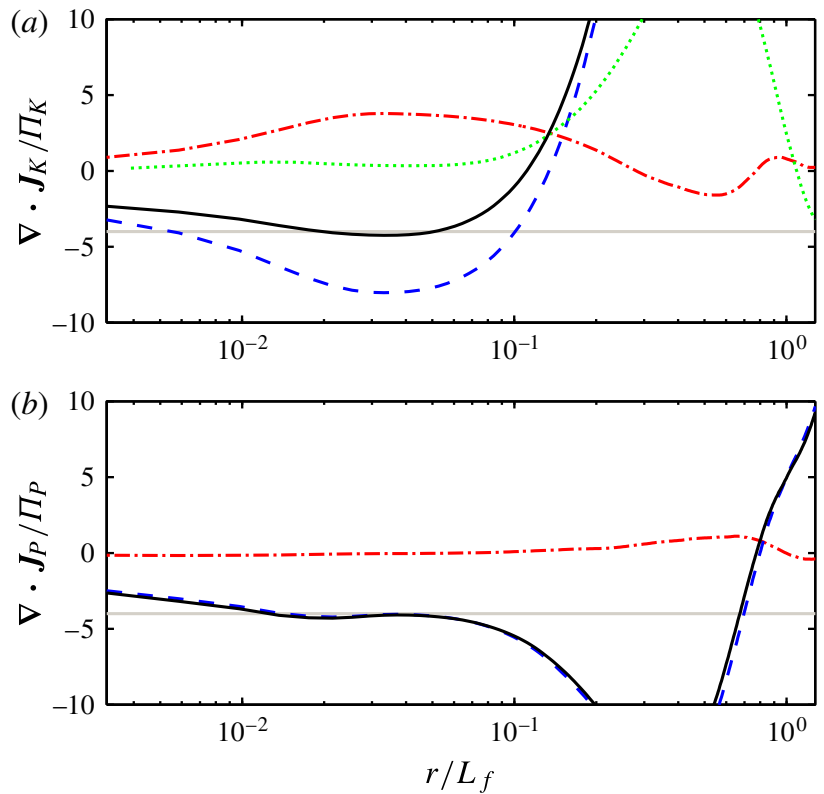

FIGURE 6. (Colour online) Decomposition of the divergence of (a) $\boldsymbol{J}_{K}=\langle\delta \boldsymbol{v} \delta \boldsymbol{u} \cdot \delta \boldsymbol{u}\rangle$ and $(b) \boldsymbol{J}_{P}=\langle\delta \boldsymbol{v} \delta b \delta b\rangle / N^{2}$ into the horizontal contribution $\left(-_{-}-{ }_{-}\right)$and the vertical contribution $(-\cdot-)$ for run Q1. Also shown are the sum of the horizontal and vertical contributions (-) and the conversion from APE to KE $2\langle\delta b \delta w\rangle(\cdots \cdots \cdots)$. Grey lines indicate the theoretical prediction -4 .

respect to the vertical resolution by decreasing the number of vertical points from 1024 to 512 and 256. In all cases, a plateau with values between 0.25 and 0.6 is found. The low-resolution runs show an increase of the ratio of only few percent. On the other hand, the forcing scheme displays a stronger effect. The $Q$-runs shows a slightly larger dependence on $R o$ and diverge as the horizontal divergence vanishes. After this point, the ratio monotonically decreases and become small at large separation distance, indicating that the two-dimensional approximation would apply to a greater extent in this range of scales.

The third-order structure functions showed in figures 1 and 2 both display a switch of sign at a particular separation distance, $r_{s}$. It is interesting to note that $r_{s} \approx 400 \mathrm{~km}$ for the velocity structure function, in figure 1 , as compared to $r_{s} \approx 1000 \mathrm{~km}$ for the velocity-temperature-temperature structure function, displayed in figure 2 . In figures 4 and 5 we can see that there is a corresponding switch of sign of the structure functions calculated from the simulations. However, for the third-order structure function including buoyancy in figure $5, r_{s}$ is close to the forcing scale in all simulations, while for the velocity structure function in figure $4, r_{s}$ moves towards smaller scales with decreasing Ro. This is particularly true for the simulations with $Q$-forcing.

In order to investigate this further we have decomposed the structure functions from the simulation into geostrophic and ageostrophic components, where the geostrophic component is the projection onto the subspace carrying only potential vorticity $q$ and the ageostrophic component is the projection onto the complementary subspace (Babin et al. 1997; Deusebio et al. 2013). In figure 8 we have plotted the 


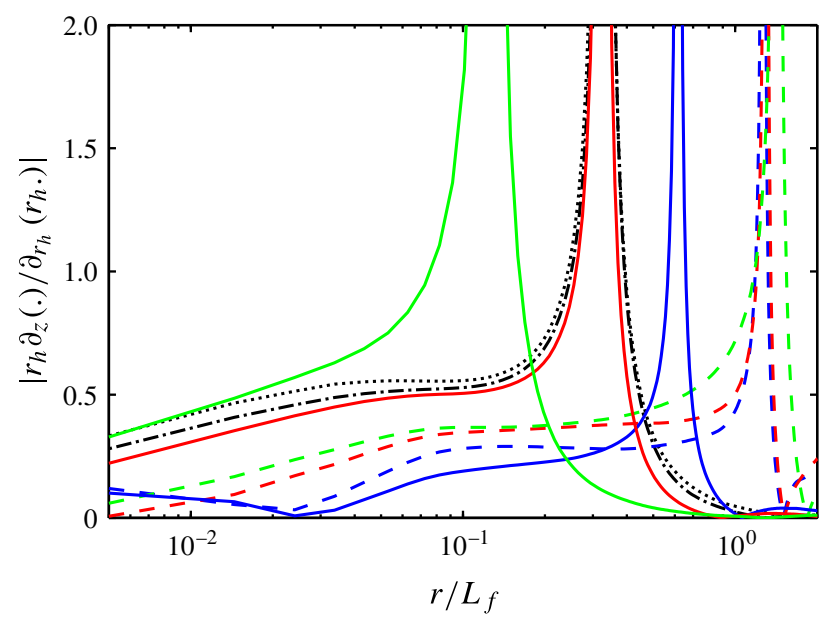

FIGURE 7. Ratio between the vertical, $\partial / \partial z\langle\delta w \delta \boldsymbol{u} \cdot \delta \boldsymbol{u}\rangle$, and the horizontal contributions $\left(1 / r_{h}\right)\left(\partial / \partial r_{h}\right)\left\langle r_{h} \delta u \delta \boldsymbol{u} \cdot \delta \boldsymbol{u}\right\rangle$ to the total divergence as a function of separation distance $r$. Coloured lines as in figure 4. Black lines refer to simulations in which the vertical resolution has been decreased to $512(-\cdot-)$ and $256(\ldots \ldots \cdots)$.
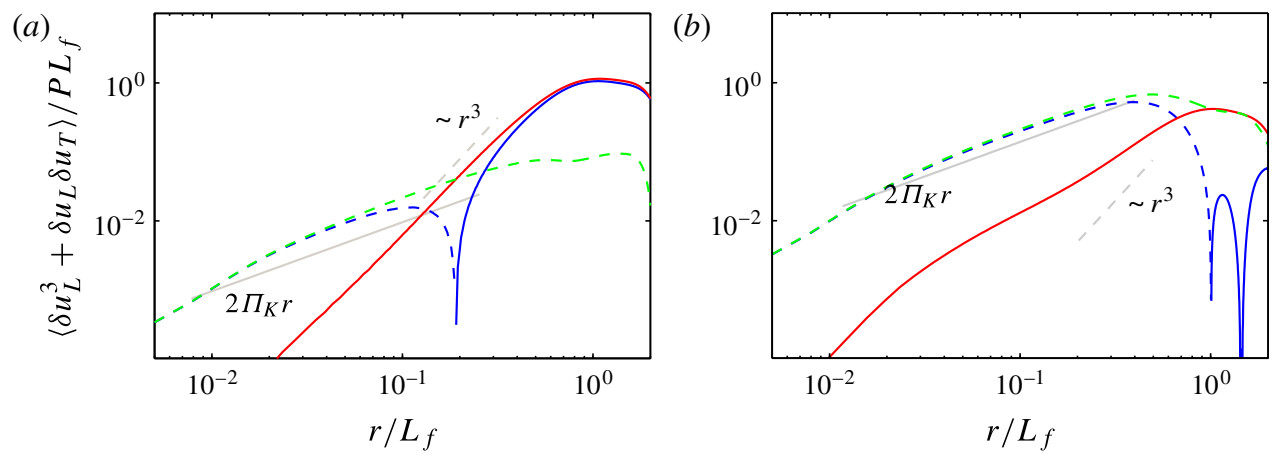

FIgURE 8. Decomposition of $\left\langle\delta u_{L} \delta \boldsymbol{u} \cdot \delta \boldsymbol{u}\right\rangle$ (blue) into its geostrophic component (red) and ageostrophic component (green). (a) Q1 and (b) M1. Solid lines: positive values; dashed lines: negative values. Grey lines are shown to aid the reader: solid, $2 \Pi_{K} r$; dashed, $\sim r^{3}$.

velocity third-order structure function from runs Q1 (figure 8a) and M1 (figure 8b) including the separate contributions from the geostrophic and the ageostrophic components. In both cases, the contribution from the geostrophic component is positive and has an almost cubic dependence on $r$, while the contribution from the ageostrophic part is negative all the way up to the forcing scale and shows a linear dependence on $r$. This is particularly true for the Q1 run where the two components have comparable magnitude. In the M1 run on the other hand, the geostrophic part is much smaller and the ageostrophic part dominates throughout the whole range of scales. In figure 9 we see the corresponding curves for the buoyancy structure function. In run Q1, both the geostrophic contribution and the ageostrophic contribution are negative up to the forcing scale, where there is a switch of sign. The geostrophic contribution shows an approximate cubic dependence while the ageostrophic contribution shows a linear dependence on $r$. In the M1 

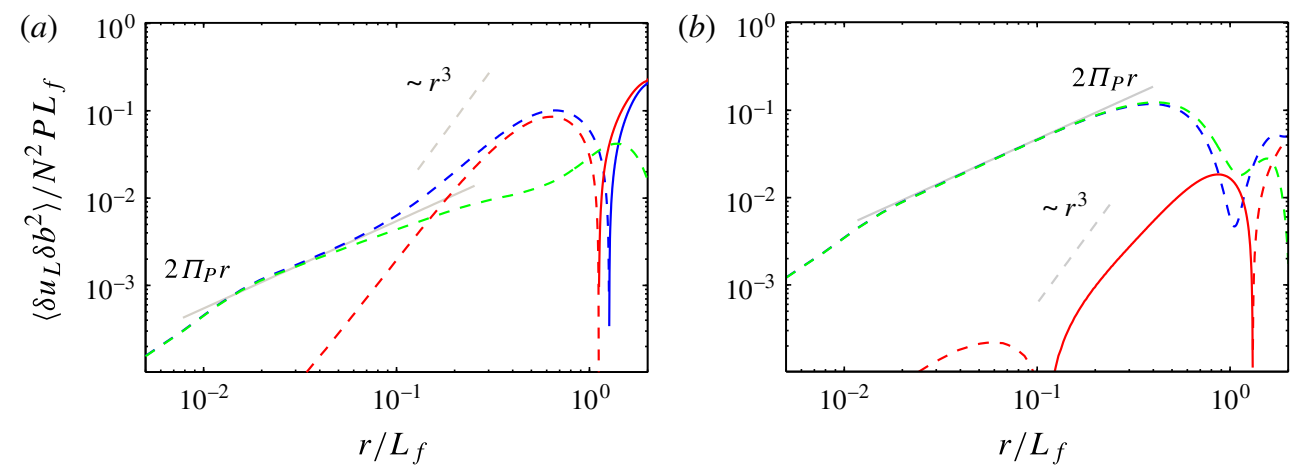

FIGURE 9. Decomposition of $\left\langle\delta u_{L} \delta b \delta b\right\rangle$ (blue) into its geostrophic component (red) and ageostrophic component (green). (a) Q1 and (b) M1. Solid lines: positive values; Dashed lines: negative values. Grey lines as in figure 8: solid, $2 \Pi_{P} r$; dashed, $\sim r^{3}$.

run, on the other hand, the geostrophic part switches sign at intermediate scales, although its magnitude is several order of magnitude smaller than the ageostrophic part. In general, the $Q$-runs are characterized by a competition between geostrophic and ageostrophic dynamics whereas the $M$-runs are dominated by the dynamics of ageostrophic modes, the latter being due to wave dynamics and/or turbulent dynamics.

In the light of these results, we may interpret the switch of sign, at $r_{s} \approx 400 \mathrm{~km}$, of the velocity structure function in figure 1 as the transition scale between a regime which is dominated by geostrophic motions and a regime which is dominated by ageostrophic motions, while the corresponding switch of sign, at $r_{s} \approx 1000 \mathrm{~km}$, of the third-order structure function in figure 2, as a signature of the forcing scale in the stratosphere. As demonstrated in the recent analysis of GCM (general circulation model) data by Augier \& Lindborg (2013), the stratosphere is mainly forced by upwards travelling planetary waves carrying energy from the troposphere, where the main forcing mechanism is baroclinic instability.

Figure 4 shows that the scale at which $\left\langle\delta u_{L} \delta \boldsymbol{u} \cdot \delta \boldsymbol{u}\right\rangle$ switches sign varies significantly, depending on the forcing scheme and on Ro. Following the concept of a 'shadow' cascade (Tung \& Orlando 2003), Vallgren et al. (2011) suggested that the transitional wavenumber $k_{s}$ can be identified as the scale at which the QG spectrum, $E_{Q G} \sim \eta^{2 / 3} k^{-3}$, with $\eta$ being the potential enstrophy dissipation, and a stratified turbulence spectrum, $E_{S T} \sim \varepsilon^{2 / 3} k^{-5 / 3}$, attain comparable magnitude, $k_{s} \sim \sqrt{\eta / \varepsilon}$, corresponding to a transitional scale $r_{s}=2 \pi / k_{s}=2 \pi \sqrt{\varepsilon / \eta}$. In figure $10(a)$ the transitional scale $r_{s}$ together with this prediction is shown for the $Q$-runs for which coexistence of a downscale enstrophy cascade and a downscale energy cascade is found (figure 10b). As figure $10(a)$ shows, the estimate of $r_{s}$ provides a fair agreement. In the $Q$-runs, the decrease of the small-scale dissipation with decreasing Ro leads to the observed reduction of $r_{s}$.

In addition to the six simulation with forcing at $k_{f}=4$ we have also carried out a simulation with forcing at $k_{f}=54$. We have done this for two reasons. First, we would like to verify that our results are not critically dependent on the forcing wavenumber. Second, we would like to investigate to what extent the upscale cascade relation (3.3) (given below) for QG turbulence is valid. Quasi-geostrophic turbulence (Charney 1971) is, in fact, very similar to two-dimensional turbulence. There are two quadratic invariants: total energy, which is the sum of KE and APE, and potential enstrophy, 

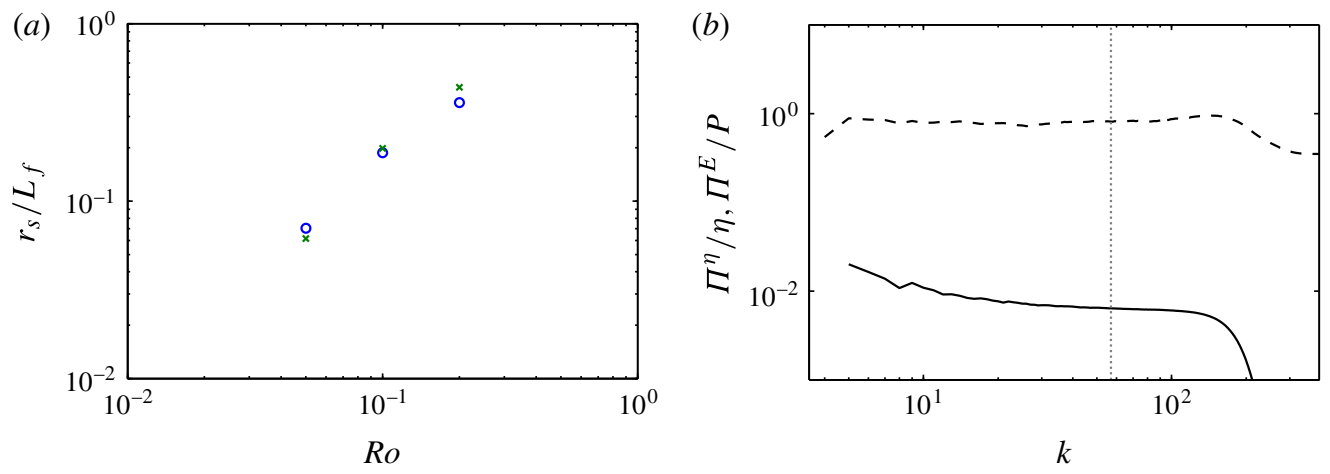

FIgURE 10. (Colour online) (a) Transition scale $r_{s}$ at which $\left\langle\delta u_{L} \delta \boldsymbol{u} \cdot \delta \boldsymbol{u}\right\rangle$ changes sign. $\bigcirc$, $Q$-runs; $\times$, prediction of the transitional scale $r_{s}=2 \pi \sqrt{\varepsilon / \eta}$ (Vallgren et al. 2011). (b) Flux of energy ( - ) and enstrophy $\left(--_{-}\right)$for the Q05 run. Vertical dotted line represents the transitional wavenumber $k_{s}=2 \pi / r_{s} \approx 50$, i.e. $k_{s} \approx 10 k_{f}$.

which is half the square of potential vorticity. Just as in two-dimensional turbulence, there are two possible cascades, a downscale cascade of potential enstrophy and an upscale cascade of energy. For the upscale energy cascade range of QG turbulence one can derive the relation (Lindborg 2007)

$$
\left\langle\delta u_{L} \delta u_{L} \delta u_{L}\right\rangle+\left\langle\delta u_{L} \delta u_{T} \delta u_{T}\right\rangle+\left\langle\delta u_{L} \delta b \delta b\right\rangle / N^{2}=-2 \Pi r
$$

where $\Pi$ is the total energy flux, which is negative since there is an upscale energy cascade. In the potential enstrophy cascade range there is no known exact third-order velocity structure function relation. However, for the enstrophy cascade range of twodimensional turbulence one can derive the exact relation (Lindborg 1999)

$$
\left\langle\delta u_{L} \delta u_{L} \delta u_{L}\right\rangle=\left\langle\delta u_{L} \delta u_{T} \delta u_{T}\right\rangle=\frac{1}{8} \Pi_{\omega} r^{3}
$$

where $\Pi_{\omega}$ is the enstrophy flux, which is positive. Cho \& Lindborg (2001) fitted the measured third-order structure function in figure 1 to a positive cubic power law in a narrow range at separation distances of the order of $1000 \mathrm{~km}$ and interpreted this range as a possible enstrophy cascade range of two-dimensional turbulence. Using the relation (3.4) and the measured third-order structure function, Cho \& Lindborg (2001) estimated the enstrophy flux as $\Pi_{\omega} \approx 2 \times 10^{-15} \mathrm{~s}^{-3}$. This interpretation seems to be far more speculative than the interpretation of the linear negative range at mesoscales as evidence of downscale energy cascade. The range with a possible cubic dependence is clearly very narrow. At $1000 \mathrm{~km}$ scales, the assumptions that such an interpretation rest on also become questionable. One such assumption is the use of Taylor's hypothesis in the analysis of the aircraft data, that is that data taken at the two different points separated by distances of a $1000 \mathrm{~km}$ may be treated as simultaneous data. Another assumption is statistical homogeneity and isotropy in the horizontal plane. However, it is worth mentioning that the value which was estimated for $\Pi_{\omega}$ is in close agreement with values estimated from GCMs (Burgess, Erler \& Shepherd 2013).

In figure 11 we plot the spectral energy flux from two simulations with the same $R o$ but with the widely different forcing wavenumbers $k_{f}=4$ and $k_{f}=54$, 


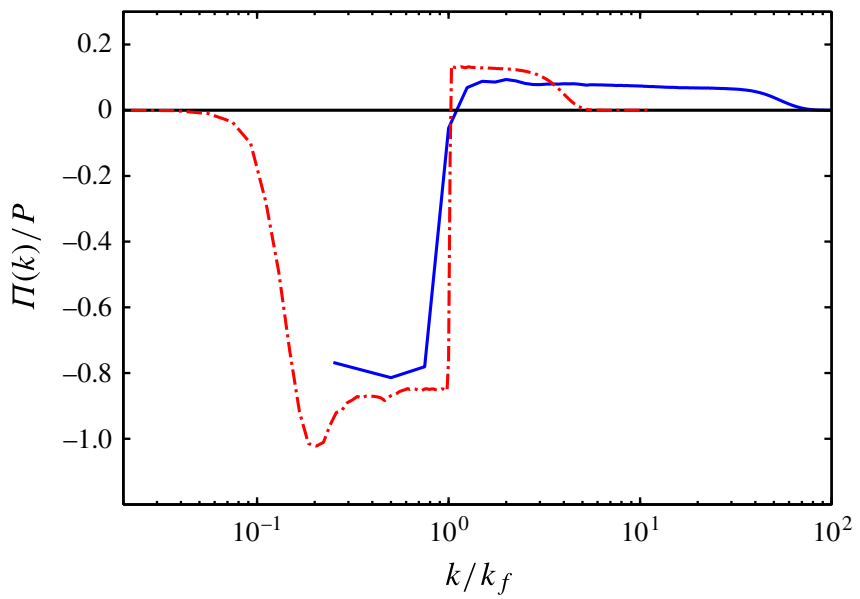

FIgURE 11. (Colour online) Total energy flux normalized with the total energy input $P$ for $R o=0.1$. - Run Q1 $\left(k_{f}=4\right) ;-\cdot-$, run Q1-SS $\left(k_{f}=54\right)$.

respectively. In both simulations, roughly one tenth of the total energy injection goes into a downscale energy cascade while the remaining part goes into an upscale energy cascade. The reason why both the upscale and downscale energy fluxes are somewhat smaller in the $k_{f}=4$ simulation is that there is some direct action of the large-scale drag at the forcing scale in this simulation. In figure 12 we show the third-order structure functions for the $k_{f}=54$ simulation. The buoyancy structure function displays a change of sign at the forcing scale, with a negative range at smaller separations and a positive range at larger separation, while the velocity structure function displays a change of sign at a smaller scale than the forcing scale. This is consistent with the results of the simulations with $k_{f}=4$. As seen in figure 12, the QG relation (3.3) is quite well satisfied in the inverse cascade range.

Cho \& Lindborg (2001) and Lindborg \& Cho (2001) calculated the transvere third-order velocity structure functions $\left\langle\delta u_{T} \delta u_{T} \delta u_{T}\right\rangle$ and $\left\langle\delta u_{T} \delta u_{L} \delta u_{L}\right\rangle$. In these studies the transverse component was taken as positive in the anticyclonic direction, that is the clockwise azimuthal component on the northern hemisphere and the anti-clockwise component on the southern hemisphere. In the following we will do it the other way around and take the transverse component as positive when it is in the cyclonic direction. With this convection, Cho \& Lindborg (2001) found that both these functions are positive and show a quadratic dependence on separation distance in the lower stratosphere. Thus, there is a preference for cyclonic motions in the lower stratosphere.

In figure 13 we have plotted the sum of the two functions, $\left\langle\delta u_{T} \delta u_{T} \delta u_{T}\right\rangle+$ $\left\langle\delta u_{T} \delta u_{L} \delta u_{L}\right\rangle$, normalized by $E_{K}^{3 / 2}$, where $E_{K}$ is the mean KE. In all simulations this function is positive and shows an approximate quadratic dependence on $r$, in agreement with the results from the stratosphere. There is no clear trend with regard to $R o$ and there is no significant difference between the simulations with $Q$-forcing and $M$-forcing. The magnitude of the curves from the simulations with $Q$-forcing is smaller, but this is due to the normalization, since $E_{K}$ is larger in these simulations. In figure 14 we have plotted the skewness $\left\langle\delta u_{T}^{3}\right\rangle /\left\langle\delta u_{T}^{2}\right\rangle^{3 / 2}$ from the simulations and also inserted a curve which is calculated based on the results given by Lindborg \& Cho (2001). For the atmospheric data, we have normalized $r$ using $L_{f}=1000 \mathrm{~km}$. As seen in figure 14, the skewness corresponding to the 


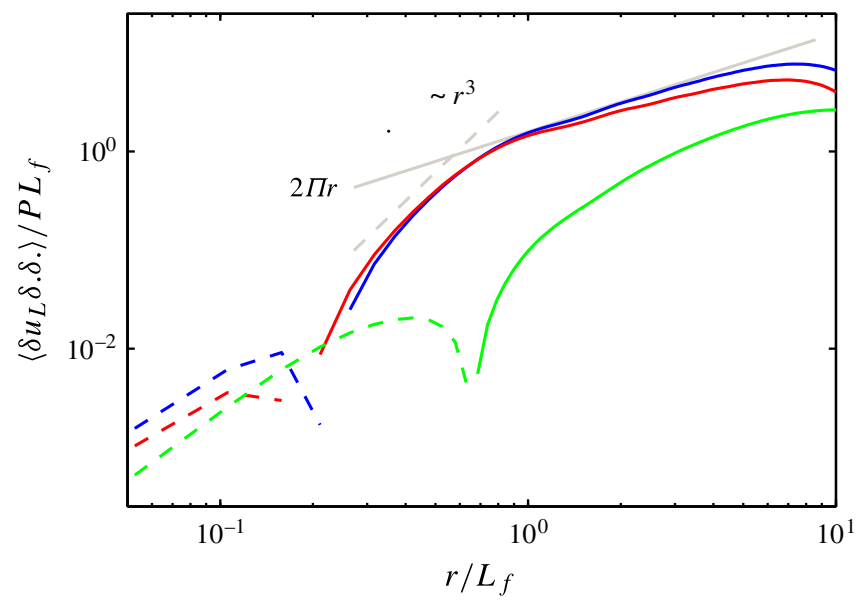

Figure 12. Plot of $\boldsymbol{J}_{K}$ (red), $\boldsymbol{J}_{P}$ (green) and $\boldsymbol{J}_{K}+\boldsymbol{J}_{P}$ (blue) for run Q1-SS $\left(k_{f}=54\right)$ : - , positive values; - - - , negative values. Solid grey line, (3.3); dashed grey line to aid the reader, $\sim r^{3}$.

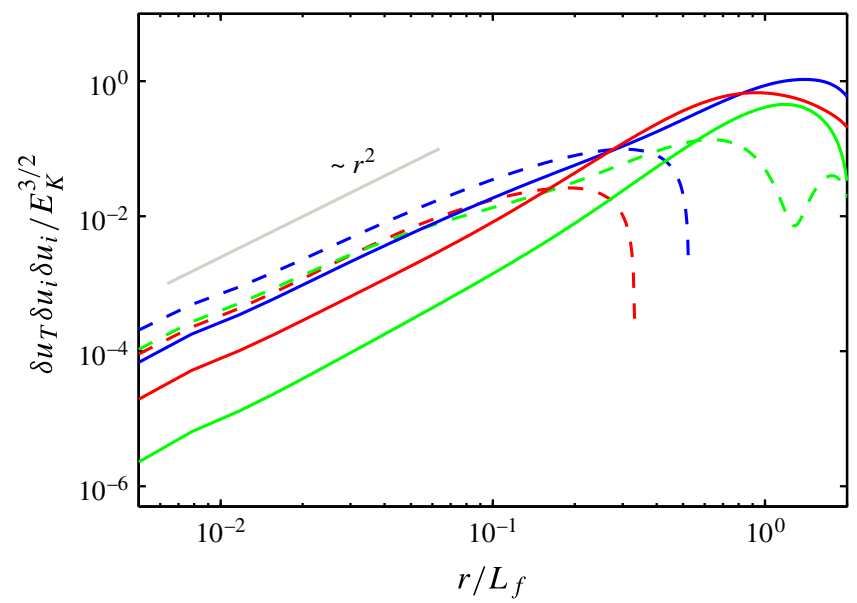

FIgURE 13. Plot of $\left\langle\delta u_{T} \delta u_{L}{ }^{2}+\delta u_{T}{ }^{3}\right\rangle$ for all of the simulations. Lines as in figure 4. Line to aid the reader, $\sim r^{2}$.

stratospheric measurements is a little bit larger than unity, indicating that there is a strong dominance of cyclonic motions, while it is lower, $\sim 0.1-0.5$, in the simulations. We can offer no theoretical explanation of these results, but only point out that there is both an interesting similarity between the observations and the simulations, in that there is a preference for cyclonic motions and that the curves show a quadratic dependence on $r$, and a striking dissimilarity, in that the magnitude of the skewness is considerably larger for the stratospheric data compared to the simulations.

\section{Conclusions}

Cho \& Lindborg (2001) measured the third-order velocity structure functions using aircraft data in the lower stratosphere. They found that $\left\langle\delta v_{L} \delta v_{L} \delta v_{L}\right\rangle+\left\langle\delta v_{L} \delta v_{T} \delta v_{T}\right\rangle$ has a negative linear dependence on $r$ in the mesoscale range and interpreted this as a sign of a downscale cascade of KE. They used the two-dimensional relation 


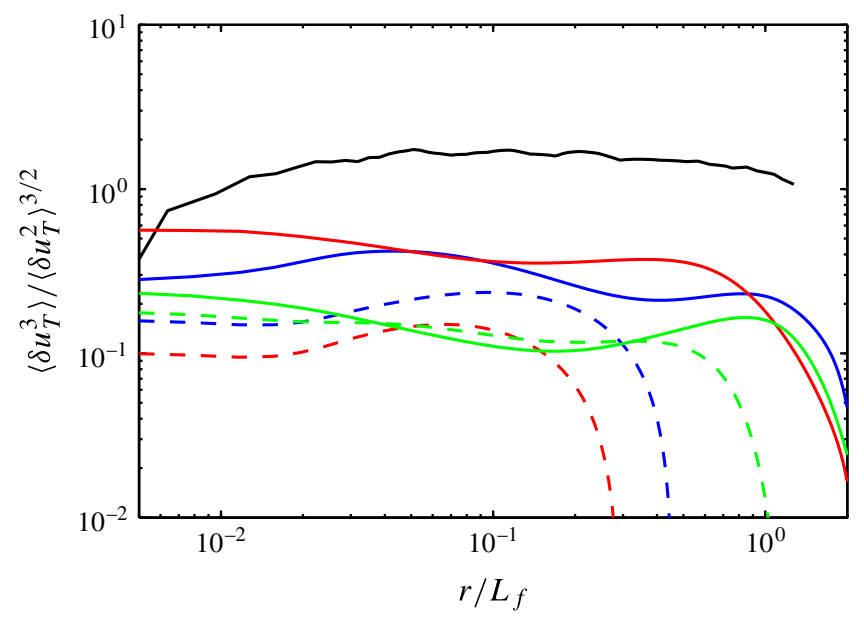

FIgURE 14. Plot of skewness $\left\langle\delta u_{T}{ }^{3}\right\rangle /\left\langle\delta u_{T}{ }^{2}\right\rangle^{3 / 2}$ for the different simulations. Coloured lines as in figure 4; black line: measurements in the atmosphere (evaluated from Cho \& Lindborg 2001). The separation distance for the atmospheric data has been normalized using $L_{f}=1000 \mathrm{~km}$.

(1.9) to estimate the spectral $\mathrm{KE}$ flux, $\Pi_{K}$, through the scales. In this paper we have suggested that the negative linear third-order velocity-temperature-temperature structure function measured by Lindborg \& Cho (2000) may be interpreted as a sign of a downscale cascade of APE. Using the two-dimensional flux relation (1.10), the spectral energy flux of $\mathrm{APE}, \Pi_{P}$, may be estimated. In order to investigate to what extent the interpretation of the measurements can be valid we have carried out idealized simulations of rotating and stratified turbulence at different Rossby numbers, using two different forcing schemes. In all simulations, the method used by Cho \& Lindborg (2001) gives a correct prediction of the sign of $\Pi_{K}$. However, the magnitude is overestimated by a factor of two. The reason for this is that the term including the vertical derivative in (1.7) cannot be neglected. For the interpretation of the third-order structure function including buoyancy, the use of the two-dimensional flux relation (1.10) provides a much more accurate estimate of the downscale energy flux.

We have also calculated the third-order structure functions which are not parity invariant and found that these scale as $r^{2}$, similar to what was found in the stratosphere, with a strong preference for cyclonic motions. However, the skewness of the cyclonic velocity increment, $\delta u_{T}$, is lower in the simulations than in the stratosphere. There are a number of studies of homogeneous rotating turbulence showing a preference for cyclonic motions (e.g. Hopfinger \& van Heijst 1993; Bartello, Metais \& Lesieur 1994). In these studies, the skewness of vertical vorticity, $\omega_{z}$, is generally used as a measure of the degree of symmetry breaking. In a field with an energy spectrum which is more shallow that $k^{-3}$, vorticity is a small-scale quantity. Therefore, the skewness of $\omega_{z}$ may be influenced by the small scales and may thus be Reynolds-number dependent (Moisy et al. 2011). On the other hand, the skewness of $\delta u_{T}$ is a measure of the symmetry breaking as a function of scale and is therefore more informative. We suggest that this measure should be used in order to study cyclonic/anticyclonic symmetry breaking.

The most important conclusion of our study is that it seems justified to interpret the measured third-order structure functions in the lower stratosphere as giving evidence 
of a downscale energy cascade of KE and APE, which is similar to what is seen in the simulations. In particular, we think that the very clean observational result for the velocity-temperature-temperature structure function, in figure 2, together with the corresponding result from the simulations, in figure 5, provides support for interpreting this as a result of the downscale APE flux, allowing us to find the estimate of $\Pi_{P} \approx 2 \times 10^{-5} \mathrm{~m}^{2} \mathrm{~s}^{-3}$. Had the same third-order structure functions as measured by Cho \& Lindborg (2001) displayed a positive sign or a dependence on $r$ far from linear in our simulations, then there would be no justification for interpreting the measurements of Cho \& Lindborg (2001) as evidence of a downscale energy cascade.

Cho \& Lindborg (2001) also measured the third-order structure functions in the upper troposphere. However, in this case it was difficult to obtain any consistent and well-converged results to be interpreted in the light of turbulence theory. There are probably two reasons why the results from the upper troposphere were less clean. First, the Brunt-Väisälä frequency shows a rather significant variation with height in the upper troposphere (Charney \& Drazin 1961), which may invalidate the assumption of statistical homogeneity in the vertical direction. Second, the release of latent heat plays a more important role in the upper tropospheric dynamics than in the stratospheric dynamics. In addition to this, one may also speculate that the difference of the forcing mechanism leads to some difference in the cascade dynamics. The troposphere is mainly forced by baroclinic instability, while the stratosphere is forced by upwards propagating planetary waves. The lower stratosphere seems to be an almost ideal test bed for theories on rotating and stratified turbulence. Generally, the Brunt-Väisälä frequency shows a small variation with height, so that statistical homogeneity is fairly well satisfied in the vertical direction and the release of latent heat is of minor importance. It is our hope that the present study will inspire more observational work on rotating and stratified turbulence in the lower stratosphere.

\section{Acknowledgement}

Computer time was provided by Swedish National Infrastructure for Computing (SNIC) which is gratefully acknowledged.

\section{REFERENCES}

Augier, P., Billant, P. \& Chomaz, J. M. 2013 Stratified turbulence forced with columnar dipoles. Numerical study. J. Fluid Mech. (submitted).

Augier, P., Billant, P., Negretti, M. E. \& Chomaz, J. M. 2014 Experimental study on stratified turbulence forced with columnar dipoles. Phys. Fluids 26, 046603.

Augier, P., Galtier, S. \& Billant, P. 2012 Kolmogorov laws for stratified turbulence. J. Fluid Mech. 709, 659-670.

Augier, P. \& LindBorg, E. 2013 A new formulation of the spectral energy budget of the atmosphere, with application to two high-resolution general circulation models. J. Atmos. Sci. 70 (70), 2293-2308.

Babin, A., Mahalov, A., Nicolaenko, B. \& Zhou, Y. 1997 On the asymptotic regimes and the strongly stratified limit of rotating Boussinesq equations. J. Theor. Comput. Fluid Dyn. 9 (3-4), 223-251.

BARTELlo, P. 1995 Geostrophic adjustment and inverse cascades in rotating stratified turbulence. J. Atmos. Sci. 52, 4410-4428.

Bartello, P., Metais, O. \& Lesieur, M. 1994 Coherent structures in rotating three-dimensional turbulence. J. Fluid Mech. 273, 1-30. 
Billant, P. \& Chomaz, J.-M. 2001 Self-similarity of strongly stratified inviscid flows. Phys. Fluids 13 (6), 1645-1651.

Brethouwer, G., Billant, P., Lindborg, E. \& Chomaz, J.-M. 2007 Scaling analysis and simulation of strongly stratified turbulent flows. J. Fluid Mech. 585, 343-368.

Burgess, B. H., ERler, A. R. \& Shepherd, T. G. 2013 The troposphere-to-stratosphere transition in kinetic energy spectra and nonlinear spectral fluxes as seen in ECMWF analyses. J. Atmos. Sci. 70 (2), 669-687.

Canuto, C., Hussaini, M. Y., Quarteroni, A. \& Zang, T. A. 1988 Spectral Methods in Fluid Dynamics. Springer.

Charney, J. G. 1971 Geostrophic turbulence. J. Atmos. Sci. 28 (6), 1087-1094.

Charney, J. G. \& Drazin, P. G. 1961 Propagation of planetary-scale disturbances from the lower into the upper atmosphere. J. Geophys. Res. 66 (1), 83-109.

Cho, J. Y. N. \& LindborG, E. 2001 Horizontal velocity structure functions in the upper troposphere and lower stratosphere: 1. Observations. J. Geophys. Res. 106 (D10), 10223-10232.

Deusebio, E., Vallgren, A. \& Lindborg, E. 2013 The route to dissipation in strongly stratified and rotating flows. J. Fluid Mech. 720, 66-103.

Dewan, E. M. 1979 Stratospheric wave spectra resembling turbulence. Science 204 (4395), 832-835.

Embid, P. F. \& MAJDA, A. J. 1998 Low Froude number limiting dynamics for stably stratified flow with small or finite Rossby numbers. J. Geophys. Astrophys. Fluid Dyn. 87 (1-2), 1-50.

Hopfinger, E. J. \& VAn Heisst, G. J. F. 1993 Vortices in rotating fluids. Annu. Rev. Fluid Mech. 25, 241-289.

Kolmogorov, A. N. 1941a Dissipation of energy in locally isotropic turbulence. Dokl. Akad. Nauk SSSR 32, 19-21 (in Russian).

Kolmogorov, A. N. $1941 b$ The local structure of turbulence in incompressible viscous fluid for very large Reynolds numbers. Dokl. Akad. Nauk SSSR 30, 299-303 (in Russian).

Kraichnan, R. H. 1967 Inertial ranges in two-dimensional turbulence. Phys. Fluids 10 (7), $1417-1423$.

Laval, J. P., McWilliams, J. C. \& Dubrulle, B. 2003 Forced stratified turbulence: Successive transitions with Reynolds number. Phys. Rev. E 68, 036308.

LILLY, D. K. 1983 Stratified turbulence and the mesoscale variability of the atmosphere. J. Atmos. Sci. 40 (3), 749-761.

LINDBORG, E. 1999 Can the atmospheric kinetic energy spectrum be explained by two-dimensional turbulence? J. Fluid Mech. 388, 259-288.

LindBorg, E. 2005 The effect of rotation on the mesoscale energy cascade in the free atmosphere. Geophys. Res. Lett. 32, L01809.

Lindborg, E. 2006 The energy cascade in a strongly stratified fluid. J. Fluid Mech. 550, 207-242.

LINDBORG, E. 2007 Horizontal wavenumber spectra of vertical vorticity and horizontal divergence in the upper troposphere and lower stratosphere. J. Atmos. Sci. 64, 1017-1025.

Lindborg, E. \& Brethouwer, G. 2007 Stratified turbulence forced in rotational and divergent modes. J. Fluid Mech. 586, 83-108.

LindboRG, E. \& CHO, J. Y. N. 2000 Determining the cascade of passive scalar variance in the lower stratosphere. Phys. Rev. Lett. 85 (26), 5663-5666.

LindBoRG, E. \& CHO, J. Y. N. 2001 Horizontal velocity structure functions in the upper troposphere and lower stratosphere: 2. Theoretical considerations. J. Geophys. Res. 106 (D10), 10233-10241.

LOREnZ, E. N. 1955 Available potential energy and the maintenance of the general circulation. Tellus 7 (2), 157-167.

Moisy, F., Morize, C., Rabaud, M. \& Sommeria, J. 2011 Decay laws, anisotropy and cycloneanticyclone asymmetry in decaying rotating turbulence. J. Fluid Mech. 666, 5-35.

Nastrom, G. D. \& GAGE, K. S. 1985 A climatology of atmospheric wavenumber spectra of wind and temperature observed by commercial aircraft. J. Atmos. Sci. 42, 950-960.

Riley, J. J. \& DEBRUYnKops, S. M. 2003 Dynamics of turbulence strongly influenced by buoyancy. Phys. Fluids 15 (7), 2047-2059. 
Smith, L. M. \& Waleffe, F. 2002 Generation of slow large scales in forced rotating stratified turbulence. J. Fluid Mech. 451, 145-168.

Tung, K. K. \& ORlando, W. W. 2003 The $k^{-3}$ and $k^{-5 / 3}$ energy spectrum of atmospheric turbulence: quasigeostrophic two-level model simulation. J. Atmos. Sci. 60 (6), 824-835.

Vallgren, A., Deusebio, E. \& Lindborg, E. 2011 A possible explanation of the atmospheric kinetic and potential energy spectra. Phys. Rev. Lett. 99, 99-101.

VAllis, G. K. 2006 Atmospheric and Oceanic Fluid Dynamics: Fundamentals and Large-Scale Circulation (Electronic Version). Cambridge University Press.

Waite, M. L. \& BARTello, P. 2004 Stratified turbulence dominated by vortical motion. J. Fluid Mech. 517, 281-308.

Waite, M. L. \& Bartello, P. 2006 The transition from geostrophic to stratified turbulence. J. Fluid Mech. 568, 89-108.

WAite, M. L. \& SNYDER, C. 2009 The mesoscale kinetic energy spectrum of a baroclinic life cycle. J. Atmos. Sci. 66, 883-901.

WAite, M. L. \& SNYDeR, C. 2013 Mesoscale energy spectra of moist baroclinic waves. J. Atmos. Sci. 70 (4), 1242-1256.

Xia, H., Byrne, D., Falkovich, G. \& Shats, M. 2011 Upscale energy transfer in thick turbulent fluid layers. Nat. Phys. 7 (4), 321-324. 